\title{
P01-8-4 Poster session
}

\section{Dehydroepiandrosterone induces Temozolomide Resistance through Attenuating DNA Damage under the Control of Sp1 Phosphorylation and Deacetylation in Glioblastoma}

\author{
Tsung-I Hsu, Jian-Ying Chuang, Wen-Chang Chang \\ Center for Neurotrauma and Regeneration, Taipei Medical University, Taiwan
}

Glioblastoma is the most malignant brain tumor without efficiently therapeutic strategy. Improvement of patient prognosis by the combined therapy of radiation with temozolomide (TMZ) is restricted within a small window due to the higher prevalence of recurrence. In particular, $\mathrm{O}^{6}$-methylguanine-DNA methyltransferase (MGMT)-mediated DNA repair is well defined as a characteristic of TMZ resistance, but MGMT-negative glioblastoma still develops an unknown mechanism to counteract TMZ-induced apoptosis. Therefore, the mechanisms underlying the resistance of glioblastoma in response to TMZ-mediated chemotherapy remains controversial. Previously, we clarified that aberrantly activated cytochrome P450 17A1-mediated neurosteroidogenesis caused TMZ resistance in MGMT-deficient glioblastoma through increasing the secretion of dehydroepiandrosterone (DHEA), which maintains the health of neurons and astrocytes in addition to being an adrenal gland-secreted steroid hormone. However, how DHEA alters the response of glioblastoma to TMZ has not been studied. In this study, we found that DHEA prevented TMZ-induced apoptosis through attenuating DNA damage characterized by the comet assay, apurinic/apyrimidinic site detection and $\gamma$ H2Ax expression in MGMTdeficient glioblastoma. In addition, DHEA activated the Lyn-Akt cascade to induce Sp1 phosphorylation which localized in TMZ-damaged DNA and was responsible to prevent DNA damage. Furthermore, p-Sp1 was deacetylated through the recruitment of $\mathrm{HDAC1} / 2$, and deacetylated Sp1 recruited proliferating cell nuclear antigen (PCNA) to attenuating DNA damage. To confirm whether DHEA-induced cellular process contributes to TMZ resistance, we established TMZresistant glioblastoma cells, A172-R. In addition to phosphorylation and deacetylation, the association of Sp1 with HDAC1/2 and PCNA was identified in A172-R, not in wild-type A172 cells. Based on these findings, we conclude that DHEA induces TMZ resistance in glioblastoma through inducing phospho-Sp1-mediated DNA repair. 